PACS: $12.20 .-\mathrm{m}, 13.40 .-\mathrm{f}, 13.60 .-\mathrm{Hb}, 13.88 .+\mathrm{e}$

\title{
EFFECTS OF SCALAR BOSON IN ELASTIC PROTON-ELECTRON SCATTERING
}

\author{
A.G. Gakh \\ V.N. Karazin Kharkiv National University \\ 4 Svobody Sq., Kharkov, 61022, Ukraine \\ e-mail:agakh@karazin.ua \\ Recieved 02, May, 2018
}

The differential cross section for the elastic scattering of unpolarized protons on unpolarized electrons at rest is calculated taking into account two mechanisms: one-photon and scalar-boson exchange. The spin correlation coefficients, when the proton beam and the electron target are both arbitrarily polarized, have also been calculated. These observables are calculated in terms of the proton electromagnetic form factors, namely magnetic and electric ones. Some peculiarities of the inverse kinematics (the mass of the colliding particle is larger than mass of the target particle) have been discussed. It was shown that all the spin correlation coefficients in the elastic proton electron collisions are proportional to the proton magnetic form factor. The same behaviour takes place for the spin correlation coefficients in the elastic electron proton scattering (the electron beam and proton target are both polarized). It was shown that only the interference of the two mechanisms (one-photon and one-boson) gives nonzero contribution to the spin correlation coefficients. If the spin vectors of the proton beam and electron target lie in the reaction plane then the corresponding spin correlation coefficients are zero for the case when scattered electron momentum is in the direction of the proton beam momentum.

KEY WORDS: polarization phenomena, electron, nucleon, form factors, inverse kinematics, scalar boson

\section{ЕФЕКТИ СКАЛЯРНОГО БОЗОНА У ПРУЖНОМУ ПРОТОН-ЕЛЕКТРОННОМУ РОЗСІЯННІ \\ А.Г. Гах}

Харківський наиіональний університет імені В.Н. Каразіна

61022, Украӥна, м. Харків, пл. Свободи, 4

Обчислене диференційний переріз для пружного розсіювання неполяризованих протонів на неполяризованих електронах у спокою з врахуванням двох механізмів: обміну одним фотоном та скалярним бозоном. Також обчислені коефіцієнти кореляції спінів, коли протонний пучок та електронна мішень обидві довільно поляризовані. Ці спостережувані обчислені у термінах протонних електромагнітних формфакторів, а саме магнітних та електричних. Обговорені деякі особливості інверсної кінематиці (маса нелітаючої частинки більше ніж маса частинки мішені). Показано, що усі коефіцієнти кореляції спінів у пружному протон електронному зіткненні пропорційні магнітному формфактору протона. Така ж поведінка має місце для коефіцієнтів кореляції спінів у пружному електрон протонному розсіянні (електронний пучок та протон мішені обидві поляризовані). Показано, що тільки інтерференція двох механізмів (однофотонного і однобозонного) надають ненульовий внесок до коефіцієнтів кореляції спінів. Якщо вектора спінів протонного пучка та електронної мішені належать до площини реакції, то відповідні коефіцієнти кореляції спінів рівні нулю у випадку, коли імпульс розсіяного електрона співпадає з напрямком імпульсу протонного пучка.

КЛЮЧОВІ СЛОВА: поляризаційні явища, електрон, нуклон, формфактори, інверсна кінематика, скалярний бозон

\section{ЭФФЕКТЫ СКАЛЯРНОГО БОЗОНА В УПРУГОМ ПРОТОН-ЭЛЕКТРОННОМ РАССЕЯНИИ A.Г. Гах}

Харьковский национальный университет им. В.Н. Каразина 61022, Украина, г. Харьков, пл. Свободы, 4

Вычислено дифференциальное сечение для упругого рассеяния неполяризованных протонов на покоящихся неполяризованных электронах с учетом вклада двух механизмов: однофотонного обмена и обмена скалярным бозоном. Также вычислены коэффициенты корреляции спинов, когда произвольно поляризованы как протонный пучок, так и электронная мишень. Эти наблюдаемые вычислены в терминах протонных электромагнитных формфакторов, а именно магнитного и электрического. Обсуждены некоторые особенности инверсной кинематики (масса налетающей частицы больше массы частицы мишени). Показано, что все коэффициенты корреляции спинов в упругом протон электронном столкновении пропорциональны магнитному формфактору протона. Такое же поведение имеет место для коэффициентов корреляции спинов в упругом электрон протонном рассеянии (электронный пучок и протонная мишень оба поляризованы). Показано, что только интерференция двух механизмов (однофотонного и однобозонного) дают ненулевой вклад в коэффициенты корреляции спинов. Если вектора спинов протонного пучка и электронной мишени лежат в плоскости реакции, то соответствующие коэффициенты корреляции спинов раны нулю в случае, когда импульс рассеянного электрона совпадает с направлением импульса протонного пучка.

КЛЮЧЕВЫЕ СЛОВА: поляризационные явления, электрон, нуклон, формфакторы, инверсная кинематика, скалярный бозон

As is known, some experimental discoveries, such as the neutrino oscillation, the existence of the dark matter (its nature and interaction are unknown today) and some astrophysical data, lead to the necessity of the consideration the physics beyond the Standard Model (SM) (see the reviews [1 -3]). The searches for the particles which are predicted in the models beyond the SM are ongoing at various laboratories (such as JLab, B factories, LHC). The author of the paper [4] reviews the current statuses of the theoretical and experimental activity about the search for low-mass scalars, pseudoscalars, and gauge bosons which appears in the beyond SM physics. 
Some models which introduce new particles, in particular scalar boson, can be motivated by various anomalies: the excess in the muon anomalous magnetic moment, the proton charge radius puzzle [5] and others. The theoretical and experimental consequences of these models are considered in [3]. The production of weakly coupled scalar bosons via nuclear de-excitation of an excited element into the ground state was discussed in [6]. The possibility that a new interaction between muons and protons is responsible for the discrepancy between the CODATA value of the proton radius and the value deduced from the measurement of the Lamb shift in muonic hydrogen was explored in [7]. The cross section and some polarization observables of elastic lepton-nucleon scattering caused by one-photon and onescalar-boson exchange have been calculated in [8]. In the paper [9] it was shown that spin-0 Dark Matter particles annihilating into electron positron pair could be responsible for the bright $511 \mathrm{keV}$ gamma ray observed by INTEGRAL [10] from the galactic bulge. A recent suggestion proposes that the observed lack of the asymptotic behavior of the pion-photon transition form factor might be due to the production of new particles or states. Two classes of models are considered. In the first, scalar or pseudoscalar particles are introduced with a mass within $10 \mathrm{MeV}$ [11]. The authors of the paper [12] propose to perform the spectroscopy of the mass structure of the rich and complex dark sectors via monophoton searches at low-energy lepton colliders.

The unpolarized and polarized observables for the elastic scattering of a proton projectile on an electron target at rest were derived in [13]. The authors of the paper [14] suggested that proton elastic scattering on atomic electrons may allow a precise measurement of the proton charge radius. The main advantage of this proposal is that inverse kinematics allows one to access very small values of the transferred momenta, up to four orders of magnitude smaller than the ones presently achieved, where the cross section is huge. The model-independent QED radiative corrections to the differential cross section of the elastic scattering of the protons on electrons at rest have been calculated in [15]. The radiative corrections due to the emission of virtual and real (soft and hard) photons in the electron vertex as well the vacuum polarization are taken into account. The possibility to build beam polarimeters for high-energy polarized proton beams on the basis of the elastic scattering of the protons on electrons at rest have been discussed in [16]. It was shown that polarimeter based on the elastic proton electron scattering gives a good opportunity to reliably measure as the longitudinal proton beam polarization as transverse. The inverse kinematics was proposed to measure neutron capture cross section of unstable isotopes [17]. For proton and alpha-induced reactions it was suggested to employ a radioactive ion beam hitting a proton or helium target at rest.

In this paper we consider the elastic scattering of protons by electrons at rest. The differential cross section for the elastic scattering of protons on electrons at rest is calculated taking into account the one photon and scalar boson exchange. The spin correlation coefficients, when the proton beam and the electron target are both arbitrarily polarized, have also been calculated. These observables are calculated in terms of the proton electromagnetic form factors.

Purpose of our research is to calculate the differential cross section for the elastic scattering of unpolarized protons on unpolarized electrons at rest taking into account two mechanisms: one-photon and scalar-boson exchange. The spin correlation coefficients, when the proton beam and the electron target are both arbitrarily polarized, have also been calculated.

\section{GENERAL FORMALISM}

Let us consider the reaction (Figure)

$$
p\left(p_{1}\right)+e^{-}\left(k_{1}\right) \rightarrow p\left(p_{2}\right)+e^{-}\left(k_{2}\right),
$$

where the particle momenta are indicated in parenthesis, and $q=k_{1}-k_{2}=p_{2}-p_{1}$ is the transferred four momentum. We consider this reaction in the lowest order, i. e., the interaction between electron and proton is described by exchange of a scalar boson or photon.

\section{Inverse kinematics}

One can show that, for a given energy of the proton beam, the maximum value of the four momentum transfer squared, in the scattering on electrons at rest, is

$$
\left(-k^{2}\right)_{\max }=\frac{4 m^{2}|\vec{p}|^{2}}{M^{2}+2 m E+m^{2}}
$$

where $\mathrm{m}(\mathrm{M})$ is the electron (proton) mass, $E(\vec{p})$ is the energy (momentum) of the proton beam.

The four momentum transfer squared is expressed as a function of the energy of the scattered electron, $\varepsilon_{2}$, as: $k^{2}=\left(k_{1}-k_{2}\right)^{2}=2 m\left(m-\varepsilon_{2}\right)$, where

$$
\varepsilon_{2}=m \frac{(E+m)^{2}+|\vec{p}|^{2} \cos ^{2} \theta_{e}}{(E+m)^{2}-|\vec{p}|^{2} \cos ^{2} \theta_{e}},
$$

where $\theta_{e}$ is the angle between the proton beam and the scattered electron momenta. 

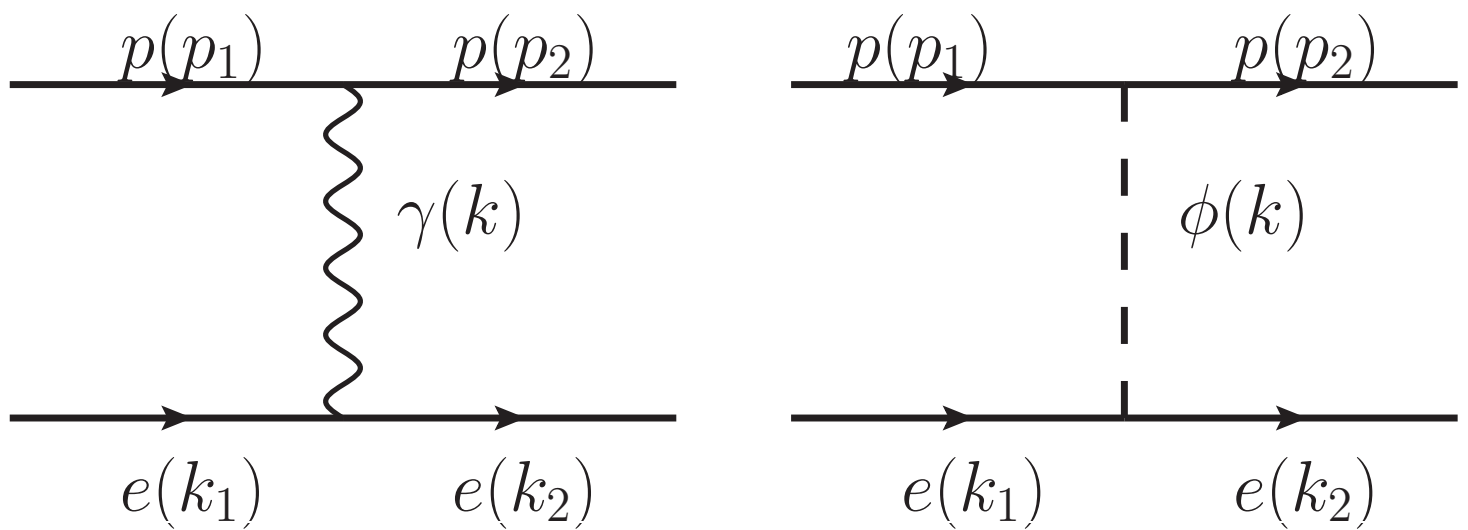

Figure. The Feynman diagrams for the reaction $p\left(p_{1}\right)+e\left(k_{1}\right) \rightarrow p\left(p_{2}\right)+e\left(k_{2}\right)$. The transfer momentum of the virtual

$$
\text { photon or scalar boson is } k=k_{1}-k_{2}=p_{2}-p_{1}
$$

From the energy and momentum conservation, one finds the following relation between the angle and the energy of the scattered electron:

$$
\cos \theta_{e}=\frac{(E+m)\left(\varepsilon_{2}-m\right)}{\left|\vec{p} \| \vec{k}_{2}\right|},
$$

where $\vec{k}_{2}$ is the momentum of the recoil electron and this formula shows that $\cos \theta_{e} \geq 0$ (the electron can never be scattered backward). One can see from Eq. (3) that, in the inverse kinematics, the available kinematical region is reduced to small values of $\varepsilon_{2}$ :

$$
\varepsilon_{2, \max }=m \frac{2 E(E+m)+m^{2}-M^{2}}{M^{2}+2 m E+m^{2}},
$$

which is proportional to the electron mass. From the momentum conservation, on can find the following relation between the energy and the angle of the scattered proton $E_{2}$ and $\theta_{p}$ :

$$
E_{2}^{ \pm}=\frac{(E+m)\left(M^{2}+m E\right) \pm M|\vec{p}|^{2} \cos \theta_{p} \sqrt{\frac{m^{2}}{M^{2}}-\sin ^{2} \theta_{p}}}{(E+m)^{2}-|\vec{p}|^{2} \cos ^{2} \theta_{p}},
$$

and this relation shows that, for one proton angle, there may be two values of the proton energies, (and two corresponding values for the recoil-electron energy and angle as well as for the transferred momentum $k^{2}$ ). This is a typical situation when the center-of-mass velocity is larger than the velocity of the projectile in the center of mass, where all the angles are allowed for the recoil electron. The two solutions coincide when the angle between the initial and final hadron takes its maximum value, which is determined by the ratio of the electron and scattered hadron $\operatorname{masses} M_{h}, \sin \theta_{h, \max }=m / M_{h}$.

\section{DIFFERENTIAL CROSS SECTION}

In the considered approximation, the matrix element $M$ of the reaction (1) can be written as a sum of two terms

$$
M=M_{\gamma}+M_{\phi}
$$

where the first (second) term describes the $\gamma(\phi)$ exchange mechanism.

Matrix element $M_{\gamma}$ can be written as:

$$
M_{\gamma}=\frac{e^{2}}{k^{2}} j_{\mu} J_{\mu}
$$

where $j_{\mu}\left(J_{\mu}\right)$ is the leptonic (hadronic) electromagnetic current. The leptonic current is

$$
j_{\mu}=\bar{u}\left(k_{2}\right) \gamma_{\mu} u\left(k_{1}\right)
$$

where $u\left(k_{1,2}\right)$ is the spinor of the incoming (outgoing) electron. The hadronic electromagnetic current can be written as 


$$
\begin{gathered}
J_{\mu}=\bar{u}\left(p_{2}\right)\left[F_{1}\left(k^{2}\right) \gamma_{\mu}-\frac{1}{2 M} F_{2}\left(k^{2}\right) \sigma_{\mu \nu} k_{v}\right] u\left(p_{1}\right)= \\
\bar{u}\left(p_{2}\right)\left[G_{M}\left(k^{2}\right) \gamma_{\mu}-F_{2}\left(k^{2}\right) P_{\mu}\right] u\left(p_{1}\right),
\end{gathered}
$$

where $F_{1}\left(k^{2}\right)$ and $F_{2}\left(k^{2}\right)$ are the Dirac and Pauli proton electromagnetic form factors, $G_{M}\left(k^{2}\right)=F_{1}\left(k^{2}\right)+F_{2}\left(k^{2}\right)$ is the Sachs proton magnetic form factor, $P_{\mu}=\left(p_{1}+p_{2}\right)_{\mu} /(2 M)$, and $M$ is the proton mass.

Matrix element $M_{\phi}$ can be written as:

$$
M_{\phi}=\frac{g_{e} g_{p}}{k^{2}-m_{\phi}^{2}} \bar{u}\left(p_{2}\right) u\left(p_{1}\right) \bar{u}\left(k_{2}\right) u\left(k_{1}\right)
$$

where $m_{\phi}$ is the scalar boson mass and the interaction between the scalar boson and electron or proton has the following form: $g_{i} \phi \bar{u}_{i} u_{i}$, (i=e, p), $g_{i}$ is the corresponding coupling constant, $\phi$ is the wave function of the scalar boson.

The matrix element squared is written as

$$
M^{2}=16 \pi^{2} \frac{\alpha^{2}}{k^{4}}\left(A_{\gamma}+\lambda A_{\gamma \phi}+\lambda^{2} A_{\phi \phi}\right)
$$

where

$$
A_{\gamma}=L_{\mu v} W_{\mu v}, L_{\mu v}=j_{\mu} j_{v}^{*}, W_{\mu v}=J_{\mu} J_{v}^{*}, \lambda=\frac{k^{2}}{e^{2}} \frac{g_{e} g_{p}}{k^{2}-m_{\phi}^{2}},
$$

and $\alpha=e^{2} /(4 \pi)=1 / 137$ is the electromagnetic fine structure constant.

The leptonic tensor, $L_{\mu \nu}$ for unpolarized initial and final electrons has the form:

$$
L_{\mu \nu}^{(0)}=2 k^{2} g_{\mu \nu}+4\left(k_{1 \mu} k_{2 v}+k_{1 v} k_{2 \mu}\right) \text {. }
$$

The contribution to the electron tensor corresponding to the polarized electron target is

$$
L_{\mu \nu}^{(p)}=2 i m \varepsilon_{\mu v \alpha \beta} k_{\alpha} S_{\beta},
$$

where $S_{\beta}$ is the initial electron polarization four vector.

The hadronic tensor, $W_{\mu \nu}^{(0)}$ for unpolarized initial and final protons can be written in the standard form, through two unpolarized structure functions:

$$
W_{\mu \nu}^{(0)}=\left(-g_{\mu \nu}+\frac{k_{\mu} k_{v}}{k^{2}}\right) W_{1}\left(k^{2}\right)+P_{\mu} P_{v} W_{2}\left(k^{2}\right) .
$$

The structure functions $W_{i}, i=1,2$, are expressed in terms of the nucleon electromagnetic form factors

$$
W_{1}\left(k^{2}\right)=-2 k^{2} G_{M}^{2}\left(k^{2}\right), W_{2}\left(k^{2}\right)=8 M^{2} \frac{G_{E}^{2}\left(k^{2}\right)+\tau G_{M}^{2}\left(k^{2}\right)}{1+\tau},
$$

where $G_{E}\left(k^{2}\right)=F_{1}\left(k^{2}\right)-\tau F_{2}\left(k^{2}\right)$ is the proton electric form factor and $\tau=-k^{2} / 4 M^{2}$.

The second term in Eq. (12) has the following form

$$
\begin{gathered}
A_{\gamma \phi}=-2 \operatorname{Re}\left\{\operatorname{Sp}\left[u\left(k_{2}\right) \bar{u}\left(k_{2}\right) \gamma_{\mu} u\left(k_{1}\right) \bar{u}\left(k_{1}\right)\right] \operatorname{Sp}\left[u\left(p_{2}\right) \bar{u}\left(p_{2}\right) B_{\mu} u\left(p_{1}\right) \bar{u}\left(p_{1}\right)\right]\right\}, \\
B_{\mu}=\left[G_{M}\left(k^{2}\right) \gamma_{\mu}-F_{2}\left(k^{2}\right) P_{\mu}\right] .
\end{gathered}
$$

The third term in Eq. (12) is

$$
A_{\phi \phi}=\operatorname{Sp}\left[u\left(k_{2}\right) \bar{u}\left(k_{2}\right) u\left(k_{1}\right) \bar{u}\left(k_{1}\right)\right] \operatorname{Sp}\left[u\left(p_{2}\right) \bar{u}\left(p_{2}\right) u\left(p_{1}\right) \bar{u}\left(p_{1}\right)\right] .
$$


The differential cross section is related to the matrix element squared by

$$
d \sigma=\frac{(2 \pi)^{4} \overline{|M|^{2}}}{4 \sqrt{\left(k_{1} \cdot p_{1}\right)^{2}-m^{2} M^{2}}} \frac{d^{3} \vec{k}_{2}}{(2 \pi)^{3} 2 \varepsilon_{2}} \frac{d^{3} \vec{p}_{2}}{(2 \pi)^{3} 2 E_{2}} \delta^{4}\left(k_{1}+p_{1}-k_{2}-p_{2}\right),
$$

where $p_{2}\left(E_{2}\right)$ is the momentum (energy) of the final proton, $\varepsilon_{2}$ is the energy of the scattered electron and the bar denotes averaging over the spins of the proton beam and electron target. From this point, formulas will differ from the elastic electron-proton scattering, as we introduce a reference system where the electron is at rest. In this system, the differential cross section can be written as:

$$
\frac{d \sigma}{d \varepsilon_{2}}=\frac{1}{32 \pi} \frac{|\bar{M}|^{2}}{m \vec{p}^{2}}
$$

where $\vec{p}$ is the momentum of the proton beam. Using the relation $k^{2}=2 m\left(m-\varepsilon_{2}\right)$ one can write

$$
\frac{d \sigma}{d k^{2}}=\frac{1}{64 \pi} \frac{\bar{M}^{2}}{m^{2} \vec{p}^{2}}
$$

The differential cross section over the solid angle can be written as

$$
\frac{d \sigma}{d \Omega_{e}}=\frac{1}{32 \pi^{2}} \frac{1}{m p} \frac{\vec{k}_{2}^{3}}{\left(-k^{2}\right)} \frac{\bar{M}^{2}}{E+m},
$$

where $d \Omega_{e}=2 \pi d \cos \theta_{e}$ (due to azimuthal symmetry). We used the relation

$$
d \varepsilon_{2}=\frac{p}{E+m} \frac{\vec{k}_{2}^{3}}{m\left(\varepsilon_{2}-m\right)} \frac{d \Omega_{e}}{2 \pi} .
$$

The expression of the differential cross section for unpolarized proton-electron scattering (averaged over the initial electron and proton spins), in the coordinate system where the electron is at rest can be written as:

$$
\frac{d \sigma}{d k^{2}}=\frac{\pi \alpha^{2}}{2 m^{2} \vec{p}^{2}} \frac{D}{k^{4}}, \quad D=\left(D_{\gamma}+\lambda D_{\gamma \phi}+\lambda^{2} D_{\phi \phi}\right)
$$

where

$$
\begin{gathered}
D_{\gamma}=k^{2}\left(k^{2}+2 m^{2}\right) G_{M}^{2}\left(k^{2}\right)+2\left[k^{2} M^{2}+\frac{1}{1+\tau}\left(2 m E+\frac{k^{2}}{2}\right)^{2}\right]\left[G_{E}^{2}\left(k^{2}\right)+\tau G_{M}^{2}\left(k^{2}\right)\right], \\
D_{\gamma \phi}=-4 m M G_{E}\left(k^{2}\right)\left(4 m E+k^{2}\right), \quad D_{\phi \phi}=4 M^{2}(1+\tau)\left(2 m^{2}-k^{2}\right) .
\end{gathered}
$$

\section{POLARIZATION OBSERVABLES}

Let us consider the spin correlation coefficients when both initial particles have arbitrary polarization, $\vec{p}+\vec{e} \rightarrow p+e$. These polarization observables were considered in [16], in view of using the polarized proton-electron scattering for the measurement of the longitudinal and transverse polarizations of a high energy proton beams.

Let us calculate the hadronic tensor, when the initial proton is polarized. The contribution of the proton polarization to the hadronic tensor is:

$$
W_{\mu \nu}\left(\eta_{1}\right)=-2 i G_{M}\left(k^{2}\right)\left[M G_{M}\left(k^{2}\right) \varepsilon_{\mu v \alpha \beta} k_{\alpha} \eta_{1 \beta}-F_{2}\left(k^{2}\right) P \cdot \eta_{1} \varepsilon_{\mu v \alpha \beta} p_{1 \alpha} p_{2 \beta}\right],
$$

where the four vector $\eta_{1}$ stands for initial proton polarization. One can see that all the correlation coefficients in $\vec{p} \vec{e}$ collisions are proportional to the proton magnetic form factor. This is a well known fact for $\overrightarrow{e p}$ scattering [18]. The dependence of the different polarization observables, namely the spin correlation coefficients, on the polarization four vector of the initial proton is completely determined by the spin dependent part of the hadronic tensor $W_{\mu \nu}\left(\eta_{1}\right)$. 
Let us choose an orthogonal system with the $z$ axis directed along the proton beam momentum $\vec{p}$, scattered electron momentum $\vec{k}_{2}$ lies in the $x z$ plane and the $y$ axis is directed along the vector $\vec{p} \times \vec{k}_{2}$. Therefore, in this system $\ell\|z, t\| x$ and $n \| y$.

In the considered frame, where the target electron is at rest, the polarization four vector of the initial proton (electron) has the following components

$$
\eta_{1}=\left(\frac{\vec{p} \cdot \vec{S}_{1}}{M}, \vec{S}_{1}+\frac{\vec{p}\left(\vec{p} \cdot \vec{S}_{1}\right)}{M(E+M)}\right), S=(0, \vec{\xi}),
$$

where $\vec{S}_{1}(\vec{\xi})$ is the unit vector describing the polarization of the initial proton (electron) in its rest system.

Applying the P-invariance of the hadron electromagnetic interaction, one can write the following expression for the dependence of the differential cross section on the polarization of the initial particles:

$$
\frac{d \sigma}{d k^{2}}\left(\vec{\xi}, \vec{S}_{1}\right)=\left(\frac{d \sigma}{d k^{2}}\right)_{u n}\left[1+C_{\ell \ell} \xi_{\ell} S_{1 \ell}+C_{t t} \xi_{t} S_{1 t}+C_{n n} \xi_{n} S_{1 n}+C_{\ell t} \xi_{\ell} S_{1 t}+C_{t \ell} \xi_{t} S_{1 \ell}\right]
$$

where $C_{i k}, i, k=\ell, t, n$ are the corresponding spin correlation coefficients which characterize elastic proton-electron scattering in the case when the proton beam and electron target are both arbitrarily polarized.

The spin correlation coefficients can be written as follows

$$
C_{i j}=C_{i j}^{(\gamma)}+\lambda C_{i j}^{(\gamma \phi)}+\lambda^{2} C_{i j}^{(\phi \phi)},
$$

where $i, j=\ell, t, n$.

The expressions of the spin correlation coefficients due to the $\gamma$ exchange mechanism are

$$
\begin{gathered}
D C_{n n}^{(\gamma)}=4 m M k^{2} G_{E}\left(k^{2}\right) G_{M}\left(k^{2}\right), \\
D C_{t t}^{(\gamma)}=4 m M \tau k^{2} \frac{G_{M}\left(k^{2}\right)}{1+\tau}\left[\left(1-\frac{4 M^{2}}{k_{\max }^{2}}\right) G_{E}\left(k^{2}\right)+\left(\frac{k^{2}}{k_{\max }^{2}}-1\right) G_{M}\left(k^{2}\right)\right], \\
D C_{t \ell}^{(\gamma)}=8 m M p\left[-k^{2}\left(1-\frac{k^{2}}{k_{\max }^{2}}\right)\right]^{1 / 2} \frac{G_{M}\left(k^{2}\right)}{1+\tau}\left\{\tau\left[G_{M}\left(k^{2}\right)-G_{E}\left(k^{2}\right)\right]-\right. \\
\left.-\frac{k^{2}}{k_{\max }^{2}} \frac{m(E+m)}{s}\left[\tau G_{M}\left(k^{2}\right)+G_{E}\left(k^{2}\right)\right]\right\}, \\
D C_{\ell t}^{(\gamma)}=-2 m M \frac{k^{2}}{p}\left(\frac{E}{M}-\frac{M}{m}\right)\left[-k^{2}\left(1-\frac{k^{2}}{k_{\max }^{2}}\right)\right]^{1 / 2} \frac{G_{M}\left(k^{2}\right)}{1+\tau}\left[\tau G_{M}\left(k^{2}\right)+G_{E}\left(k^{2}\right)\right], \\
D C_{\ell \ell}^{(\gamma)}=4 k^{2} \frac{G_{M}\left(k^{2}\right)}{1+\tau}\left\{\left(m E-\tau M^{2}\right) G_{E}\left(k^{2}\right)+\tau\left(M^{2}+m E\right) G_{M}\left(k^{2}\right)-\right. \\
\left.-\left(M^{2}+m E\right) \frac{k^{2}}{k_{\max }^{2}} \frac{m(E+m)}{s}\left[\tau G_{M}\left(k^{2}\right)+G_{E}\left(k^{2}\right)\right]\right\},
\end{gathered}
$$

where $s=M^{2}+m^{2}+2 E m$.

The expressions of the spin correlation coefficients due to the interference of the $\gamma$ and scalar boson exchange mechanisms are 


$$
\begin{gathered}
D C_{n n}^{(\gamma \phi)}=-4 G_{M}\left(k^{2}\right)\left\{E^{2} k_{2}^{2}+(E+m) \frac{k^{2}}{m}\left[E \varepsilon_{2}+\frac{k^{2}}{4 m}(E+m)\right]\right\}, \\
D C_{t t}^{(\gamma \phi)}=-4(E+m) G_{M}\left(k^{2}\right)\left[E k_{2}^{2}+E \varepsilon_{2} \frac{k^{2}}{m}+(E+m)\left(1-\frac{m E}{p^{2}}\right) \frac{k^{4}}{4 m^{2}}\right], \\
D C_{t \ell}^{(\gamma \phi)}=2 G_{M}\left(k^{2}\right) \frac{M}{p} k_{2} k^{2}(E+m) \sin \theta_{e}, \\
D C_{\ell t}^{(\gamma \phi)}=4 k_{2} G_{M}\left(k^{2}\right) \sin \theta_{e}\left[p E\left(m+\varepsilon_{2}\right)+\frac{k^{2}}{2 m}(E+m)\left(p+m \frac{E}{p}\right],\right. \\
D C_{\ell \ell}^{(\gamma \phi)}=-\frac{1}{M} G_{M}\left(k^{2}\right)\left\{4 E\left(M^{2} k_{2}^{2}+2 p^{2} \varepsilon_{2}^{2}\right)+2 \frac{k^{2}}{m}(E+m)\left[4 E^{2} \varepsilon_{2}+m E\left(k_{2}-\varepsilon_{2}\right) \frac{k^{2}}{2 m} M^{2}\right]+\right. \\
\left.+\frac{1}{p^{2}} \frac{k^{4}}{m^{2}}(E+m)^{2}\left(m M^{2}+2 E p^{2}\right)\right\} .
\end{gathered}
$$

And at last we have

$$
D C_{i j}^{(\phi \phi)}=0, \quad i, j=\ell, t, n .
$$

\section{CONCLUSION}

The elastic scattering of protons from electrons at rest was investigated in a relativistic approach in the one photon and scalar boson exchange approximation.

The differential cross section and the spin correlation coefficients, when the proton beam and the electron target are both arbitrarily polarized, have been calculated in terms of the proton electromagnetic form factors.

\section{REFERENCES}

1. Fundamental Physics at the Intensity Frontier, Report of the Workshop held December 2011 in Rockville, MD, Workshop Chairs: J.L. Hewett, and H. Weerts, arXiv:1205.2671v1 [hep-ex].

2. Essig R. et al. Dark Sectors and New, Light, Weakly-Coupled Particles // arXiv:1311.0029v1 [hep - ph].

3. Alexander Jim et al. Dark Sectors 2016 Workshop: Community Report // arXiv: 1608.08632v1 [hep - ph].

4. Soffer Abner. Searches for light scalars, pseudoscalars, and gauge bosons // arXiv: 1507.02330v1 [hep-ex].

5. Pohl et R. al. Laser spectroscopy of muonic deuterium // Science. - 2016. - Vol. 353. - P. 669-673.

6. Izaguirre Eder, Krnjaic Gordan, Pospelov Maxim. Probing New Physics with Underground Accelerators and Radioactive Sources // arXiv: 1405.4864v1 [hep-ph].

7. Tucker-Smith David, Yavin Itay. Muonic hydrogen and MeV forces // Phys. Rev. - 2011. - Vol. D83. - P.101702, arXiv: 1011.4922v1 [hep-ph].

8. Liu Yu-Sheng, Miller Gerald A. Polarized lepton-nucleon elastic scattering and a search for a light scalar boson // Phys. Rev. 2015. - Vol. C92. - P.035209, arXiv: 1507.04399v1 [nucl- th].

9. Fayet P. Light spin-1/2 or spin-0 Dark Matter particles // Phys. Rev. - 2004. - Vol. D70. - P.023514, arXiv: hep-ph /0403226v1, 2004.

10. Boehm C., Fayet P., Silk J. Light and Heavy Dark Matter Particles// Phys. Rev. - 2004. - Vol. D69. - P.101302, arXiv: hep-ph /0311143, 2004.

11. Lees J.P. et al. Search for new $\pi^{0}$-like particles produced in association with a $\tau$ - lepton pair // Phys. Rev. - 2014. Vol. D90. - P.112011, arXiv:1411.1806 [hep-ex]

12. Hochberg Yonit et al. Dark spectroscopy// arXiv: 1706.05008v1 [hep-ph].

13. Gakh G.I., Dbeyssi A., Marchand D., Tomasi-Gustafsson E., Bytev V.V. Polarization effects in elastic proton-electron scattering // Phys. Rev. - 2011. - Vol. C84. - P. 015212.

14. Gakh G.I., Dbeyssi A., Tomasi-Gustafsson E., Marchand D., Bytev V.V. Proton-electron elastic scattering and the proton charge radius // Physics of Particles and Nuclei Letters - 2013. - No.5. - P. 393-397.

15. Gakh G.I., Konchatnij M.I, Merenkov N.P. Tomasi-Gustafsson Egle. Radiative corrections to elastic proton-electron scattering measured in coincidence // Phys. Rev. 2017. - Vol. C95. -P. 055207.

16. Glavanakov I.V., Krechetov Yu.F., Potylitsyn A.P., Radutsky G.M., Tabachenko A.N., Nurushev S.B. A proton polarimeter based on the elastic pe - scattering, //Nucl. Instrum. Meth. - 1996. - Vol. A381. - P. 275 - 279.

17. Reifarth Rene, Litvinov Yuri A. Measurements of neutron-induced reaction in inverse kinematics // Phys. Rev. ST Accel. Beams. - 2014. - Vol. 17. - P. 014701, arXiv:1312.3714v1 [nucl-ex].

18. Akiezer A.I., Rekalo M.P. Hadron Electrodynamics . - Naukova Dumka: Kiev - 1977. - Chapter 4. 